\title{
SELEÇÃO DE CRITÉRIOS PARA PRIORIZAÇÃO DA MANUTENÇÃO PREVENTIVA DE TELECOMUNICAÇÕES EM PLATAFORMAS DE PETRÓLEO
}

\author{
Antonio Rodrigues da Silva Neto \\ Especialista em Docência do Ensino Superior / UCAM \\ arodrisn@gmail.com
}

Eduardo Shimoda

Doutorado em Ciência Animal / UENF

prof_shimoda@yahoo.com.br

\section{Simara Netto Martins}

simaraiff@gmail.com

Milton Erthal Junior

Doutor em Produção Vegetal

miltonerthal@hotmail.com

\section{RESUMO}

A maioria das reservas brasileiras de petróleo na atualidade encontra-se em campos marítimos (PETROBRAS, 2014), o que torna desafiadora a logística de atendimento às atividades da indústria de petróleo e gás no ambiente offshore. Os desafios se estendem a todas as áreas do conhecimento humano que fazem parte do cenário da região de produção. Naturalmente, os desafios serão proporcionalmente maiores onde há mais atividades. A Bacia de Campos, em função da sua representatividade no contexto da produção nacional de petróleo, vivencia esta realidade e uma das atividades a superar os desafios que se apresentam à cada dia é a de manutenção dos itens de telecomunicações. Com a missão de oferecer e garantir a comunicação de pessoas, aplicativos e processos às unidades que operam a mais de $100 \mathrm{Km}$ da costa, os órgãos internos atuam fortemente na melhoria dos processos de manutenção do diversificado parque de telecomunicações instalado nas plataformas. Dentre os esforços empreendidos encontra-se a mnutenção preventiva, a qual atualmente é realizada de forma cíclica, seguindo cronogramas pré-estabelecidos para cada rede ou sistema.Com o crescimento do número de plataformas e o aumento das distâncias em direção à região do pré-sal, a periodicidade dos ciclos de manutenção preventiva de telecomunicações pode ser afetada e decisões de priorização deverão ser tomadas na direção da escolha da plataforma a receber as ações de prevenção. Como efetuar esta priorização? Quais critérios deverão ser considerados ? Como estes critérios se relacionarão entre si para a tomada de decisão ? Este trabalho tem por objetivo responder a estas questões através de consultas à literatura, consultas a especialistas em telecomunicações no ambiente offshore e a subsequente aplicação da metodologia de Lawshe (1975), largamente utilizada no meio acadêmico para a seleção de itens pesquisados. Concluída esta etapa, os critérios selecionados servirão de base para aplicação de um método de Auxílio Multicirtério à Decisão no processo decisório numa próxima fase, a qual não está incluída no escopo deste artigo.

\section{Palavras-chave: Telecomunicações; Manutenção preventiva; Auxílio Multicritério à Decisão.}

\section{Introdução}

Nas atividades industriais, o interesse pelo aperfeiçoamento das práticas de manutenção é uma constante. Particularmente na indústria de petróleo e gás, a questão da manutenção se reveste de crucial importância, em função da natureza especialmente erigosa das atividades e dos graves riscos e 
prejuízos envolvidos. Conforme afirmado por Dekker (1996), onde há grande quantidade de capital investido em sistemas técnicos, haverá considerável necessidade de manutenção a ser realizada. $\mathrm{O}$ próprio autor inclui as indústrias de óleo e gás em sua afirmação.

Os investimentos neste ramo de atividades são elevados e. Além disso, o objeto de trabalho é extremamente perigoso, envolvendo substâncias altamente inflamáveis e tóxicas, trafegando ao longo das etapas do processo produtivo, sob condições severas de pressão e temperatura (Khan e Haddara, 2004). O aspecto da segurança é então acrescentado ao cenário de produção e dentre os fatores que contribuem para com os requisitos adicionais de segurança de uma plataforma de produção de petróleo está a disciplina de telecomunicações.

A interdependência entre a indústria de petróleo e gás e as redes de telecomunicações é mencionada por Heller (2001) e Duenas (2005) ao abordarem a inter-relação entre os sistemas de infraestrutura, tanto pela crescente influência no controle, na automação, na eficiência e na redução dos custos de operação, como também pela combinação dos fatores de recuperação por ocasiões dos desastres naturais.

Desta forma, a abordagem da manutenção nos sistemas de telecomunicações instalados nas plataformas de produção de petróleo da Bacia de Campos se reveste de importância, mesmo não se tratando de uma atividade fim do ramo de petróleo e gás. A maneira mais adequada para tratamento do

tema requer ações de prevenção e um dos caminhos a erem trilhados aponta na direção da manutenção preventiva, através da realização ações e detecção e prevenção de falhas, na busca de se manter os itens na condição specificada (Wang, 2002).

Vários são os fatores que podem influenciar na elaboração de um plano de manutenção preventiva, especialmente se considerarmos a diversidade de serviços e as tecnologias de telecomunicações a bordo das plataformas, além das peculiaridades do ambiente offshore. Esta multiplicidade de influências sinaliza para a adoção de técnicas de auxílio multicritério à decisão (AMD). Este trabalho objetiva identificar os critérios que devem ser adotados para priorização da plataforma de produção de petróleo a receber uma equipe de manutenção preventiva de telecomunicações.

\section{Revisão da literatura}

\subsection{Métodos de manutenção}

Conforme conceituado na norma NBR 5462 (Associação Brasileira de Normas Técnicas, 1994), manutenção é a combinação de todas as ações técnicas e administrativas, incluindo as de supervisão, destinadas a manter ou recolocar um item em um estado no qual possa desempenhar uma função requerida. Xenos (2004) define como métodos de manutenção: a manutenção corretiva, a manutenção preventiva, a manutenção preditiva, a melhoria nos equipamentos e a prevenção da manutenção, abordando também o conceito de manutenção produtiva.

A manutenção corretiva ocorre sempre após a falha ter ocorrido. A aparentemente economia por não se arcar com os custos de prevenção poderá ser desastrosa ou perigosa em função das conseqüências, sendo extremamente importante evitar que a falha ocorra durante a operação real (Wang, 2002), como é o caso da indústria de petróleo e gás. A manutenção preventiva, por sua vez, objetiva antecipar as ações de manutenção, efetuando paradas programadas, o que evita os problemas advindos das paradas indesejáveis motivadas pela falha nos equipamentos. Já a manutenção preditiva é considerada uma modalidade mais avançada da manutenção preventiva, valendo-se tanto da substituição de peças e componentes antes da expiração de sua vida útil (Xenos, 2004), como da monitoração de parâmetros indicadores de condição, tais como, temperatura, vibrações, pressão, desgaste, etc, através do emprego de sofisticados dispositivos (Chiochetta, et al., 2004). 
A melhoria nos equipamentos resgata o conceito japonês do kaisen, que significa fazer melhorias, o que no contexto da manutenção se traduz pela busca continuada da melhoria gradativa, para além das especificações originais, investigando-se exaustivamente as causas da falha e não apenas efetuando-se o retorno à operação. A prevenção da manutenção prega a aproximação da empresa com o fabricante do equipamento e consiste num conjunto de atividades que se iniciam desde a fase de projeto, objetivando a redução dos serviços de manutenção durante a etapa de operação. A manutenção produtiva como a aplicação mais adequada de todos os métodos de manutenção, com o intuito de otimizar os fatores econômicos da produção, de forma a garantir a melhor utilização dos equipamentos, maximizando sua produtividade a custos mais baixos (Xenos, 2004).

\subsection{Os sistemas de telecomunicações das plataformas da bacia de campos}

A área de exploração da Bacia de Campos é de aproximadamente $115.000 \mathrm{~km} 2$ (Carminatti et al., 2008). A região de produção está situada após a área de deslocamento das embarcações. É nesta região que se concentra grande parte da infraestrutura submarina, responsável pela produção dos campos de petróleo em operação (Fraga et al., 2003 e Ribeiro, 2012). A estes campos estão interligadas as plataformas responsáveis pela produção de petróleo.

Uma plataforma de produção de petróleo offshore consiste num complexo ambiente industrial, onde coexistem diversos sistemas operando sobre condições severas. $\mathrm{Na}$ Bacia de Campos, estas condições são agravadas em função da distância ao continente. $O$ emprego da tecnologia vem cada vez mais conciliando produtividade e segurança no meio offshore, uma vez que os sistemas eletrônicos possibilitam a operação e o monitoramento de forma remota, retirando os trabalhadores da proximidade dos locais potencialmente perigosos (Velez, 2003). Neste enriquecido cenário tecnológico os sistemas de telecomunicações tem a missão de transportar as informações de interesse do sistema de supervisão, os sinais de controle, de imagens, de sonorização, de telefonia, bem como viabilizar o tráfego de dados, tanto no âmbito da própria plataforma, como na interação com o mundo exterior à mesma (Junior, 2006).

Para o transporte de informações das plataformas offshore são utilizadas e combinadas diversas técnicas de transmissão, o que vai depender basicamente das distâncias a serem vencidas, do tipo e da quantidade de informações a serem transportadas. A figura 1 apresenta as formas de transmissão utilizadas na comunicação da plataforma com o mundo exterior, onde podem ser observadas: $(a, b)$ A comunicação via rádio, que pode ser utilizada através de ondas diretas, ondas terrestres, reflexões ionosféricas (ondas celestes) e enlaces de rádio- visibilidade, (c) links satélites e através de (d) comprimento de ondas luminosas via cabos ópticos dedicados ou acondicionados no interior de cabos elétricos (cabos eletro-ópticos). Cumpre ressaltar que, em função da dispersão geográfica, algumas plataformas de produção atuam na retransmissão dos sinais para aquelas que se encontram mais afastadas na rede de telecomunicações, sendo por isto denominadas de repetidoras.

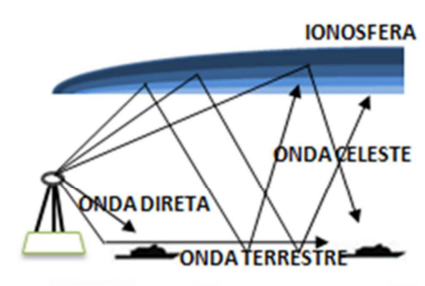

a)

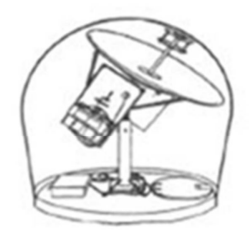

c)

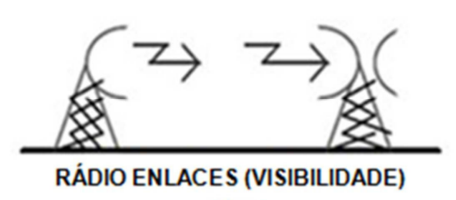

b).

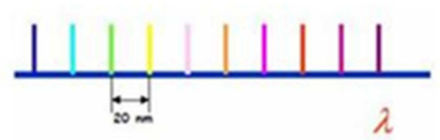

d) 
Figura 1 - Meios de transmissão a) Rádio comunicação por propagação direta, terrestre e ionosférica; b) Rádio comunicação de alta capacidade com propagação por visibilidade; c) Antena parabólica para comunicação via satélite; d) Comprimentos de ondas luminosas via Fibras ópticas. Fontes: Oliveira (2013), Marinha do Brasil e www.teleco.com.br

Tipicamente, no interior da plataforma offshore de produção de petróleo, além do sistema de transmissão encontram-se instalados os seguintes sistemas: rádio operacional, Intercomunicação industrial (conhecido como Intercom), circuito fechado de tv (CFTV), Multiplex, recepção e distribuição de TV (CATV), telefonia e rede local (PETROBRAS, 2004). Estes sistemas proporcionam serviços de telecomunicações que auxiliam os trabalhadores embarcados a executarem suas atividades operacionais, proporcionam momentos de lazer nos períodos de folga a bordo e contribuem para com os requisitos de segurança necessária, seguindo rígidos padrões internacionais (Silva, 2014). O aspecto da segurança nas operações de produção de petróleo da Bacia de Campos contempla, tanto a segurança de mar, regida pelas Normas da Autoridade Marítima - NORMAM 01(Marinha do Brasil, 2005), como a segurança de vôo de aeronaves, conforme as Instruções do Comando da Aeronáutica ICA 63-10 - (Ministério da Aeronáutica, 2008) (Dantas, 2006).

Com relação à segurança marítima, a NORMAM 01 (Marinha do Brasil, 2005), preconiza a conformidade com Sistema Global de Socorro e Segurança Marítima (GMDSS), sistema internacional que utiliza tanto comunicações terrestres como via satélites. O GMDSS norteia a comunicação a bordo das embarcações, visando garantir alerta rápido e automático nas situações de socorro marítimo e de melhorias em termos de telecomunicações entre a comunidade marítima. Emprega técnicas de automação de sistemas nas faixas de frequências do Serviço Móvel Marítimo em VHF, MF e HF,

incorporando também os sistemas Inmarsat e os satélites EPIRB. Desta forma, Obtem-se o aumento da confiabilidade e da efetividade dos sistemas de socorro e segurança em escala global. Este sistema auxilia também na disseminação das informações marítimas de segurança, o que inclui os alertas metereológicos, alertas de navegação e as previsões do tempo. Sua finalidade básica é unificar as comunicações entre as autoridades de busca e salvamento e as demais embarcações próximas da que está em perigo, quando da ocorrência de um evento indesejável, de modo a permitir operações de busca e salvamento coordenadas (ANATEL, 2014).

Quanto à segurança de vôo, a ICA 63-10 (Ministério da Aeronáutica, 2008) o cenário de comunicação da plataforma é composto basicamente pelos equipamentos de rádio comunicação com aeronaves e pelo Rádio-Farol, que provê o balizamento eletrônico, sendo ativado somente em situações restritas de auxilio à navegação aérea, mediante autorização.

Prover e integrar toda esta gama de serviços em instalações localizadas em mar aberto, a distâncias superiores a $100 \mathrm{Km}$ da costa é tarefa complexa. O mau funcionamento dos equipamentos e sistemas que suportam os serviços de telecomunicações da plataforma pode provocar impactos indesejáveis no ambiente de produção de petróleo como um todo (Junior, 2006). Uma abordagem consistente da manutenção preventiva sobre os equipamentos que compõem os sistemas de telecomunicações instalados a bordo da plataforma de produção de petróleo pode contribuir para o funcionamento harmonioso das atividades operacionais, dos momentos de descanso e lazer, com os requisitos de segurança adequados.

\section{Metodologia}

\subsection{Consulta à literatura}


Na primeira fase do trabalho foi efetuada uma consulta à literatura em meados de dezembro de 2013, com objetivo de se identificar itens relevantes a integrarem a lista primária de critérios de priorização. Para isto foi utilizada a Base Scopus, por sua importância no meio científico, procurando-se obter um mínimo de dez artigos. As palavras chaves, na língua portuguesa, utilizadas no filtro de pesquisa foram: priorização, manutenção, critérios e telecomunicações, enquanto que na língua inglesa as correspondentes palavras utilizadas foram: prioritization, maintenance, criteria and telecommunications. A busca foi estendida até os oito anos anteriores e adicionalmente, como alternativa, foi ampliada aos sítios de universidades e publicações de seminários, o que resultou num total de onze publicações.

\subsection{Consulta aos especialistas}

Concluída a etapa inicial, passou-se à fase de pesquisa exploratória com o objetivo de se colher contribuições de técnicos especializados em sistemas de telecomunicações offshore.

Para isto foi empregado um questionário aberto como instrumento de coleta de dados. O questionário foi elaborado procurando-se associar os serviços (benefícios) proporcionados pelos sistemas de telecomunicações instalados nas plataformas com a infraestrutura que integra os respectivos sistemas.

Ao todo foram entrevistados 13 especialistas com mais de 20 anos de experiência. As entrevistas foram todas realizadas diretamente no próprio ambiente de trabalho ou por e-mail. O conjunto de especialistas foi formado por profissionais da área de interligação de redes de longa distância (WAN), segurança de redes, comunicação de voz, infraestrutura, transmissão, energia, CFTV, comunicação via satélite e serviços de telecomunicações.

\subsection{Seleção dos itens}

A lista das novas contribuições sobre critérios, obtida junto aos especialistas, foi adicionada àquela construída durante a etapa de consulta à literatura e enviada para análise, por e-mail, ao orpo técnico embarcado nas plataformas de produção de petróleo. O objetivo era colher dos respondentes a opinião sobre cada critério através das seguintes alternativas: "1- não importante", "2- importante, mas não essencial" e "3- essencial" (Lawshe, 1975). O total de respondentes nesta etapa chegou a 23, correspondendo a cerca de $41 \%$ do efetivo embarcado durante o período de coleta.

Para cada critério, a quantidade dos respondentes que o consideraram "essencial" foi computada para cálculo da Razão de Validade de Conteúdo (CVR):

$$
C V R=\frac{\mathrm{n}-\frac{\mathrm{N}}{2}}{\frac{\mathrm{N}}{2}}
$$

Em que:

CVR: razão da validade de conteúdo

n: número de respondentes que consideram o critério como "essencial"

$\mathrm{N}$ : número total de respondentes

A expressão nos indica que, quanto mais acima da metade dos respondentes percebem o critério como "essencial", maior o seu grau de validade conteúdo e o resultado calculado é positivo. Por outro lado, quanto mais abaixo da metade dos respondentes percebem o critério como "essencial, menor o seu grau de validade conteúdo e o resultado calculado é negativo. Caso exatamente a metade dos respondentes perceberem o critério como "essencial, a validade de conteúdo obtém o valor zero. 
Os CVR calculados para cada critério foram comparados com seu valor crítico (CVRcrítico), à luz da tabela de validação de Lawshe (1975), posteriormente corrigida por Wilson (2012), selecionando-se os critérios cujo CVR calculado estivessem acima do respectivo CVRcrítico. A Tabela 1 apresenta os valores de CVR crítico até 23 especialistas.

Tabela 1 - Valores para CVRcrítico até 23 especialistas

\begin{tabular}{|c|c|c|c|c|c|c|c|}
\cline { 2 - 7 } \multicolumn{1}{c|}{} & \multicolumn{7}{|c|}{ Teste de significáncia unicaudal } \\
\cline { 2 - 7 } & $10 \%$ & $5 \%$ & $2,5 \%$ & $1 \%$ & $0,5 \%$ & $0,1 \%$ \\
\cline { 2 - 7 } & \multicolumn{5}{|c|}{ Teste de significáncia bicaudal } \\
\hline $\begin{array}{c}\text { Número de } \\
\text { especialistas }\end{array}$ & $20 \%$ & $10 \%$ & $5 \%$ & $2 \%$ & $1 \%$ & $0,2 \%$ \\
\hline 5 & 0,573 & 0,736 & 0,877 & 0,990 & 0,990 & 0,990 \\
\hline 6 & 0,523 & 0,672 & 0,800 & 0,950 & 0,990 & 0,990 \\
\hline 7 & 0,484 & 0,622 & 0,741 & 0,879 & 0,974 & 0,990 \\
\hline 8 & 0,453 & 0,582 & 0,693 & 0,822 & 0,911 & 0,990 \\
\hline 9 & 0,427 & 0,548 & 0,653 & 0,775 & 0,859 & 0,990 \\
\hline 10 & 0,405 & 0,520 & 0,620 & 0,736 & 0,815 & 0,977 \\
\hline 11 & 0,386 & 0,496 & 0,591 & 0,701 & 0,777 & 0,932 \\
\hline 12 & 0,370 & 0,475 & 0,566 & 0,672 & 0,744 & 0,892 \\
\hline 13 & 0,355 & 0,456 & 0,544 & 0,645 & 0,714 & 0,857 \\
\hline 14 & 0,343 & 0,440 & 0,524 & 0,622 & 0,688 & 0,826 \\
\hline 15 & 0,331 & 0,425 & 0,506 & 0,601 & 0,665 & 0,798 \\
\hline 16 & 0,320 & 0,411 & 0,490 & 0,582 & 0,644 & 0,773 \\
\hline 17 & 0,311 & 0,399 & 0,475 & 0,564 & 0,625 & 0,749 \\
\hline 18 & 0,302 & 0,388 & 0,462 & 0,548 & 0,607 & 0,728 \\
\hline 19 & 0,294 & 0,377 & 0,450 & 0,534 & 0,591 & 0,709 \\
\hline 20 & 0,287 & 0,368 & 0,438 & 0,520 & 0,576 & 0,691 \\
\hline 21 & 0,280 & 0,359 & 0,428 & 0,508 & 0,562 & 0,674 \\
\hline 22 & 0,273 & 0,351 & 0,418 & 0,496 & 0,549 & 0,659 \\
\hline 23 & 0,267 & 0,343 & 0,409 & 0,485 & 0,537 & 0,644 \\
\hline
\end{tabular}

Fonte: Freitas (2013)

\section{Resultados}

\subsection{Itens compilados da literatura}

A consulta resultou na lista de onze publicações descritas no Quadro 1, ordenadas por ano de publicação. 


\begin{tabular}{|c|c|c|}
\hline Ano & Autor(es) & Critérios extraídos \\
\hline 1995 & Rana V. S., & $\begin{array}{l}\text { Ausência de Contingência, Histórico de falhas, Sistema de } \\
\text { Energia, Sistema de recepção Satélite }\end{array}$ \\
\hline 1997 & Menezes, H. B., & Histórico de falhas \\
\hline 2002 & Wang $\mathrm{H}$ & $\begin{array}{l}\text { Custos, Histórico de falhas, Substituição de Equipamentos } \\
\text { c/ maior tempo de uso }\end{array}$ \\
\hline 2003 & Morais, V.C; Muhlen, S.C., & $\begin{array}{l}\text { Custos, Facilidade de manutenção, Facilidade de } \\
\text { Operação, Histórico de falhas, Impacto ao Negócio, } \\
\text { Importância Estratégica, Segurança, }\end{array}$ \\
\hline 2004 & $\begin{array}{l}\text { Bezerra, E. K.; Nakamura, E. T.; } \\
\text { Lima, M. B.; Ribeiro, S. L. }\end{array}$ & $\begin{array}{l}\text { Enlace rádio, GMDSS, Internet, Rádio Farol, Radio } \\
\text { operacional, Radio VHF aeronáutico, Repetidor Ativo, } \\
\text { Segurança, Serviço de Dados, Serviço de voz, Sistema de } \\
\text { recepção Satélite, Sistema Trunking }\end{array}$ \\
\hline 2006 & Cunha, A. J. B; Pinto, E. L. F & $\begin{array}{l}\text { Histórico de falhas, Quantidade de clientes, Sistema de } \\
\text { Energia }\end{array}$ \\
\hline 2007 & Klein, J.J., & Dificuldades Logísticas, Impacto ao Negócio, Segurança \\
\hline 2008 & $\begin{array}{l}\text { Marcato, A. L. M.; Faria, H. J.; } \\
\text { Brandão, L. E. T.; Pimenta A.A.; } \\
\text { Senra, P. M. A. Silveira, A. }\end{array}$ & Dificuldades Logísticas, Histórico de falhas \\
\hline 2008 & Souza, R. D. & Custos, Histórico de falhas, Impacto ao Negócio \\
\hline 2009 & $\begin{array}{l}\text { Gifhorn, E.; Ensslin, L.; Ensslin, S. } \\
\text { R. ; Vianna, W. B }\end{array}$ & $\begin{array}{l}\text { Caixas de Passagem, Conexão, Distribuição, Proteção } \\
\text { elétrica, Sistema de Energia }\end{array}$ \\
\hline 2012 & Almeida, A. T. & Custos, Histórico de falhas, Impacto ao Negócio \\
\hline
\end{tabular}

Quadro 1 - Lista de publicações analisadas. Fonte: elaboração própria

Apenas Rana (1995), Menezes (1997) e Bezerra et al (2004) abordaram diretamente a área de telecomunicações, este último contribuiu com o maior número de critérios. Os demais autores contribuíram com a abordagem da gestão da manutenção, contemplando a manutenção reventiva, fazendo alusão a critérios associados à área. Nesta análise preliminar foram elencados 25 critérios.

O critério que se mais se destacou foi Histórico de falhas, citado por 8 dentre as 11 publicações. Em seguida aparecem empatados, com 4 ocorrências cada um, Custos e Impacto no negócio, o que no caso de uma plataforma de produção de petróleo, corresponde ao impacto na sua própria produção de petróleo.

\subsection{Itens compilados a partir dos especialistas}

Os critérios obtidos a partir da consulta aos especialistas são apresentados na Figura 2, juntamente com a quantidade de indicações recebidas. 


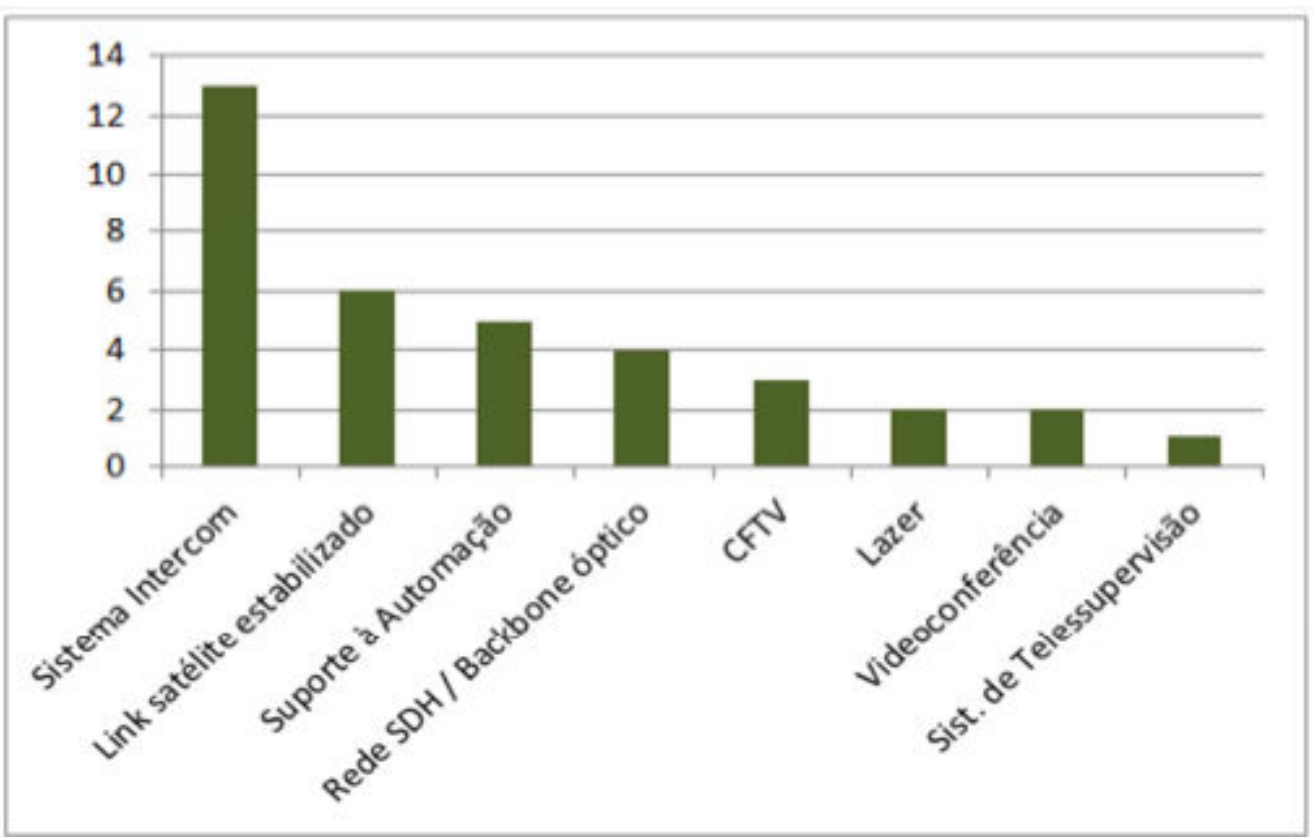

Figura 2 - Critérios acrescentados na fase de análise exploratória - Fonte: elaboração própria

Observa-se que o critério Sistema Intercom obteve a unanimidade dos especialistas consultados, o que denota a sua importância. Link satélite estabilizado apareceu em segundo lugar nas citações dos mesmos, com 6 ocorrências.

\subsection{Itens selecionados}

Os resultados obtidos pelo método de Lawshe são apresentados no Quadro 2, de onde se obtém a decisão sobre a importância ou não de cada critério. A decisão de se manter o critério ocorre quando o valor do CVR calculado está acima do valor do CVR crítico, sendo função do total de avaliadores (respondentes) do critério e da quantidade de avaliações na condição de essencial que o critério recebeu. 


\begin{tabular}{|l|l|c|c|c|c|c|c|}
\hline \hline Cód. & Critério & $\mathbf{N e}$ & $\mathbf{N}$ & $\mathbf{\%}_{\text {essencial }}$ & $\mathbf{C V R}_{\text {calc }}$ & $\mathbf{C V R}_{\text {crit }}$ & Decisão \\
\hline C1 & Ausência de Link de Contingência & 14 & 23 & $60,9 \%$ & 0,217 & 0,267 & Excluir \\
\hline C2 & CFTV & 7 & 22 & $31,8 \%$ & $-0,364$ & 0,273 & Excluir \\
\hline C3 & Custos & 6 & 20 & $30,0 \%$ & $-0,400$ & 0,286 & Excluir \\
\hline C4 & CXs de Passg, Conexão, Distribuição & 9 & 23 & $39,1 \%$ & $-0,217$ & 0,267 & Excluir \\
\hline C5 & Dificuldades Logísticas (vôo, vaga, acesso, etc) & 10 & 23 & $43,5 \%$ & $-0,130$ & 0,267 & Excluir \\
\hline C6 & Enlace rádio & 21 & 22 & $95,5 \%$ & 0,909 & 0,273 & Manter \\
\hline C7 & Equipamentos GMDSS & 18 & 22 & $81,8 \%$ & 0,636 & 0,273 & Manter \\
\hline C8 & Facilidade de manutenção & 2 & 22 & $9,1 \%$ & $-0,818$ & 0,273 & Excluir \\
\hline C9 & Facilidade de Operação & 1 & 19 & $5,3 \%$ & $-0,895$ & 0,294 & Excluir \\
\hline C10 & Histórico de falhas & 16 & 22 & $72,7 \%$ & 0,455 & 0,273 & Manter \\
\hline C11 & Impacto ao Negócio da Empresa & 19 & 23 & $82,6 \%$ & 0,652 & 0,267 & Manter \\
\hline C12 & Importância Estratégica & 20 & 23 & $87,0 \%$ & 0,739 & 0,267 & Manter \\
\hline C13 & Internet Cultural & 1 & 23 & $4,3 \%$ & $-0,913$ & 0,267 & Excluir \\
\hline C14 & Lazer & 1 & 23 & $4,3 \%$ & $-0,913$ & 0,267 & Excluir \\
\hline C15 & Link Satélite com Antena Estabilizada & 19 & 23 & $82,6 \%$ & 0,652 & 0,267 & Manter \\
\hline C16 & Proteção elétrica & 9 & 23 & $39,1 \%$ & $-0,217$ & 0,267 & Excluir \\
\hline C17 & Quantidade de clientes a bordo & 4 & 23 & $17,4 \%$ & $-0,652$ & 0,267 & Excluir \\
\hline C18 & Rádio Farol & 10 & 22 & $45,5 \%$ & $-0,091$ & 0,273 & Excluir \\
\hline C19 & Radio operacional & 9 & 22 & $40,9 \%$ & $-0,182$ & 0,273 & Excluir \\
\hline C20 & Repetidor Ativo & 18 & 21 & $85,7 \%$ & 0,714 & 0,279 & Manter \\
\hline C21 & Rede SDH-NG / Backbone óptico & 18 & 22 & $81,8 \%$ & 0,636 & 0,273 & Manter \\
\hline C22 & Segurança & 20 & 21 & $95,2 \%$ & 0,905 & 0,279 & Manter \\
\hline
\end{tabular}

\begin{tabular}{|l|l|c|c|c|c|c|c|}
\hline C23 & Serviço de Dados - Rede Integrada Corporativa & 15 & 23 & $65,2 \%$ & 0,304 & 0,267 & Manter \\
\hline C24 & Serviço de voz - Rede de Ramais internos & 15 & 23 & $65,2 \%$ & 0,304 & 0,267 & Manter \\
\hline C25 & Sistema Intercom & 23 & 23 & $100,0 \%$ & 1,000 & 0,267 & Manter \\
\hline C26 & Sistema de Energia & 22 & 23 & $95,7 \%$ & 0,913 & 0,267 & Manter \\
\hline C27 & Sistema de Telessupervisão & 6 & 23 & $26,1 \%$ & $-0,478$ & 0,267 & Excluir \\
\hline C28 & Sistema Trunking & 9 & 22 & $40,9 \%$ & $-0,182$ & 0,273 & Excluir \\
\hline C29 & Substituir Equipamentos c/ maior tempo de uso & 5 & 23 & $21,7 \%$ & $-0,565$ & 0,267 & Excluir \\
\hline C30 & Suporte à Automação & 7 & 23 & $30,4 \%$ & $-0,391$ & 0,267 & Excluir \\
\hline C31 & TVRO / CATV & 1 & 21 & $4,8 \%$ & $-0,905$ & 0,279 & Excluir \\
\hline C32 & VHF aeronáutico & 19 & 23 & $82,6 \%$ & 0,652 & 0,267 & Manter \\
\hline C33 & Videocon & 4 & 23 & $17,4 \%$ & $-0,652$ & 0,267 & Excluir \\
\hline
\end{tabular}

Quadro 2 - Aplicação de Lawshe (1975) para seleção dos critérios. Fonte: elaboração própria

A decisão sobre os critérios efetivamente validados pela aplicação do método encontra-se destacada para facilitar a identificação. Observe-se que o critério Segurança obteve a unanimidade de validação.

\section{Discussões e construção da hierarquia dos critérios}

Os critérios selecionados através da aplicação da metodologia de Lawshe (1975) são constituídos por equipamentos, sistemas, serviços, características da plataforma associadas ao negócio, à posição estratégica, além de um parâmetro típico da gestão de manutenção, o Histórico de falhas, citado por Xenos (2004) como Número de falhas. O objetivo da construção da hierarquia entre estes critérios foi identificar a existência ou não de possíveis relacionamentos, bem como a possibilidade de agrupamento entre os mesmos.

Não foi identificada nenhuma possibilidade de agrupamento ou de relação critério subcritério para os critérios Impacto ao negócio (Produção) e Importância estratégica, em conseqüência, eles integram a estrutura hierárquica para o problema na condição de critérios independentes de primeiro nível.

Com relação ao critério Histórico de falhas, observa-se um relacionamento direto deste com os critérios selecionados sob a forma de equipamentos, sistemas, redes e serviços. Visando facilitar a futura aplicação do método de Auxílio Multicritério à Decisão, este critério será utilizado como 
elemento de agrupamento, de acordo com as afinidades identificadas, de maneira a se obter a relação critério-subcritério onde possível.

A primeira relação critério-subcritério identificada está associada ao aspecto da Segurança. Observa-se que fazem parte da lista de critérios validados alguns equipamentos e sistemas introduzidos na planta de telecomunicações da plataforma, justamente para prover a segurança. Os três critérios associados à segurança validados pelos técnicos offshore abordam respectivamente: a segurança de mar (GMDSS), a segurança de vôo ( $V H F$ aeronáutico) e a segurança operacional do dia-a-dia da plataforma (Intercom). Conclui-se então que os critérios Sistema de intercom, Equipamentos GMDSS e VHF aeronáutico podem ser considerados subcritérios do critério Segurança. A reunião dos históricos de falhas destes critérios é que vai produzir o parâmetro do histórico de falhas de Segurança.

Outro agrupamento importante pode ser obtido ao se identificar a existência de serviços de telecomunicações destacados no Quadro 3. Segundo Pires e Piccinini (1997), os serviços de telecomunicações são entregues por um conjunto de equipamentos e meios de transmissão interligados de modo a suportar a interconexão da comunicação dos diferentes usuários. Estes equipamentos e meios de transmissão configuram um sistema, cuja arquitetura se dá sob a forma de redes, as quais são compostas por equipamentos. Na prática, não se executa a manutenção preventiva sobre os serviços de telecomunicações, mas sim sobre os equipamentos que oferecem estes serviços. Ao se eleger serviços como critérios para a realização da manutenção preventiva, está se afirmando implicitamente que os equipamentos que entregam estes serviços é que são efetivamente os critérios. Por conseguinte, os históricos de falhas dos equipamentos que entregam os Serviços de voz e os Serviços de dados podem ser agrupados numa relação critério-subcritério de Histórico de falhas de serviços.

Finalmente, os demais critérios demarcados no Quadro 3 possuem a característica de permearem a infraestrutura da planta de telecomunicações da plataforma, tanto do ponto de vista da energia que abastece os itens de telecom (Sistema de energia), como dos meios de comunicação: via rádio (Enlace rádio), via satélite (Antena estabilizada), via fibra óptica (Backbone óptico) e na comunicação operacional interna (Repetidor ativo). Este relacionamento integrará o critério Histórico de falhas de infraestrutura.

A Figura 3 mostra a estrutura hierárquica completa contendo os cinco critérios principais. Os critérios Importância estratégica (repetidora) e produção (Impacto ao negócio) não possuem subcritérios. Já os critérios Histórico de falhas de segurança, Histórico de falhas de Serviços e Histórico de falhas de Infraestrutura, possuem respectivamente 3, 2 e 5 subcritérios. As alternativas estão identificadas como PLAT 1 a PLAT N, representando as plataformas que farão parte do futuro processo de seleção. Esta estrutura atende ao limite máximo de 11 critérios para futura utilização do método de Análise Hierárquica (Saaty, 1991) na seleção da plataforma a ser priorizada para envio de uma equipe de manutenção preventiva de telecomunicações. 


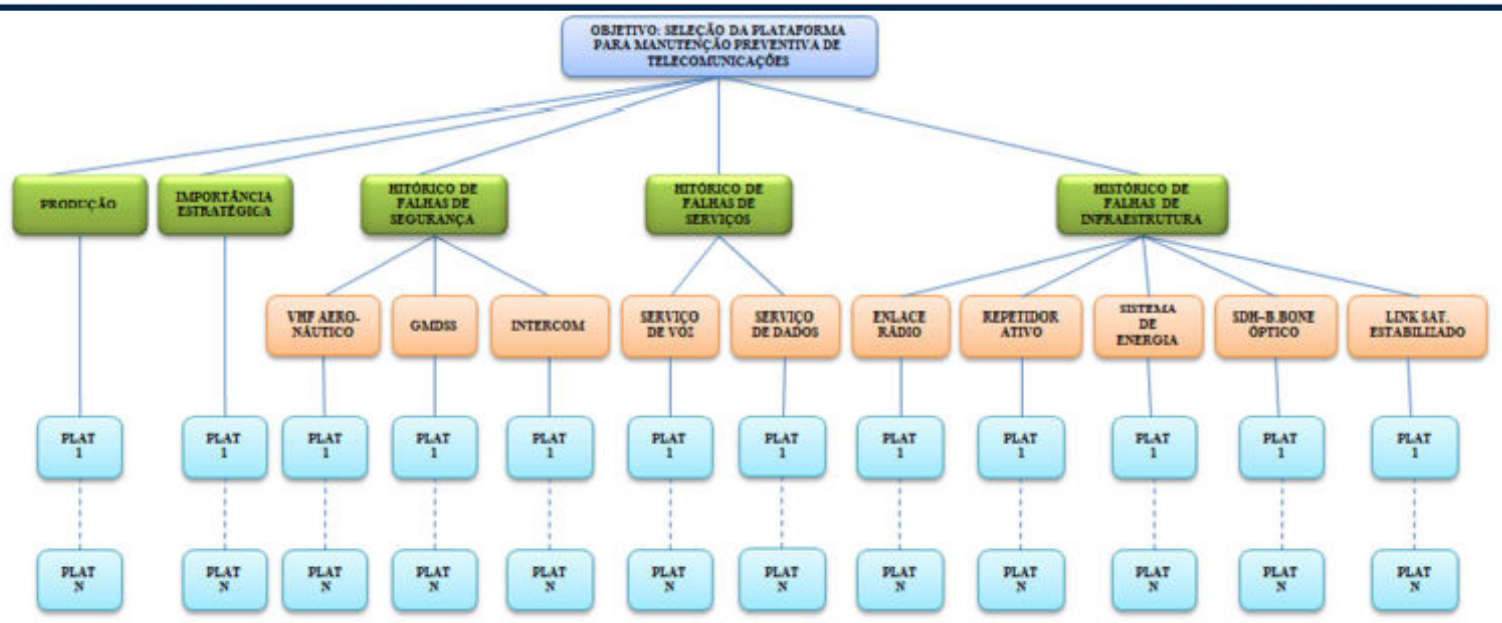

Figura 3 - Estrutura hierárquica para o problema. Fonte: elaboração própria

\section{Conclusões e considerações finais}

Este trabalho abordou a seleção de critérios de maior relevância para a escolha da plataforma de produção de petróleo a receber uma equipe de manutenção preventiva de telecomunicações. Destacou que a questão envolve a análise multicriterial, apresentando os critérios selecionados e a relação entre eles.

A seleção dos critérios utilizou tanto a busca à literatura, como a consulta a especialistas, sendo apoiada na metodologia de Lawshe (1975) amplamente utilizada no meio acadêmico.

Entre todos os parâmetros o que apresentou maior influência no que diz respeito aos aspectos técnicos específicos de telecomunicações, o histórico de falhas foi o principal destaque, possibilitando inclusive a relação entre critérios e subcritérios.

Com relação aos aspectos inerentes ao ambiente offshore, a segurança foi o que mais recebeu a atenção do técnico especializado em telecomunicações embarcado. Isto se refletiu na valoração de $100 \%$ dos respondentes e também na seleção de subcritérios relacionados à segurança de mar, à segurança de voo e à segurança interna no âmbito da plataforma.

Em termos de trabalhos futuros, sugere-se sua continuidade através da aplicação de um método de Auxílio Multicritério à Decisão e a correspondente utilização de dados reais, sob a forma de estudo de caso.

\section{REFERÊNCIAS}

ALMEIDA, A. T. Multicriteria Model for Selection of Preventive Maintenance Intervals. Wiley Online Library.

Quality and Reliability Engineering International. Vol.28, 2012, ANATEL. Sistema Global de Socorro e Segurança Maritima GMDSS - Global Maritime Distress and Safety System. Disponível em: http://www.anatel.gov.br/Portal. Acesso em: 30/06/2014.

ASSOCIAÇÃO BRASILEIRA DE NORMAS TÉCNICAS. NBR 5462: Confiabilidade e mantenabilidade. Rio

de Janeiro: ANBT, 1994. Disponível em http://www.abntcatalogo.com.br. Acesso em 30 de junho de 2014. 
BEZERRA, E. K.; NAKAMURA, E. T.; LIMA, M. B.; RIBEIRO, S. L. Proteção da Infra-estrutura crítica e

Telecomunicações análise, metodologia e aplicações. USP. São Paulo, 2004.

CARMINATTI, M.; WOLFF, B.; GAMBOA, L. New exploratory frontiers in Brazil. In: 19th World Petroleum

Congress, pp. 1-11, Madri, Espanha, 2008.

CHIOCHETTA, J. C.; HATAKEYAMA, K.; MARÇAL, R. F. M. Sistema de Gestão da Manutenção para a

Pequena e Média Empresa. In: Encontro Nacional de Engenharia de Produção. Florianópolis. 2004.

CUNHA, A. J. B; PINTO, E. L. F. Estudos para a Definição da Priorização da Manutenção. XVII Seminário

nacional de distribuição de energia elétrica. Belo Horizonte. MG, 2006.

DANTAS, L. A. Modelo de gestão baseado na conformidade legal de plataformas de petróleo operando em águas jurisdicionais brasileiras. UFRJ. Dissertação de Mestrado. 2006

DEKKER, R. Applications of maintenance optimization models: a review and analysis. Reliability Engineering and System Safety 51. 229-240. Erasmus University, Rotterdam, The Netherlands. 1996

DUEÑAS, L. A. O. Interdependent Response of Networked Systems to Natural Hazards and Intentional Disruptions. School of Civil and Environmental Engineering. Georgia Institute of Technology. 2005

FRAGA, C. T. C., BORGES, F. A., BELLOT, C., et al. Campos Basin-25 Years of Production and its Contribution to the Oil Industry. In: Offshore Technology Conference, Houston, Texas, EUA. 2003

FREITAS, R. J, Um estudo sobre critérios de avaliação de sucesso na implementação de sistemas ERP. Dissertação de Mestrado. UFF. Niterói, 2013.

GIFHORN, E.; ENSSLIN, L.; ENSSLIN, S. R. ; VIANNA, W. B. Aperfeiçoamento da gestão organizacional por meio da abordagem multicritério à decisão. Revista Gestão Industrial. UTFPR. Ponta Grossa. PR, 2009

HELLER, M. Interdependencies in Civil Infrastructure Systems. The Bridge, National Academy of Engineering. Winter, 2001. Disponível em: http://www.nae.edu. Acesso em 19/07/2014

JUNIOR, S. F. J., Utilização de novas tecnologias de telecomunicações em plataformas petrolíferas offshore. Dissertação de Mestrado. UFRN. Natal. RN, 2006.

KHAN, F.I., HADDARA, M. Risk-Based Maintenance (RBM): A new approach for process plant inspection and maintenance. Process Safety Progress. 2004

KLEIN, J.J. Desenvolvimento e implantação de um sistema de planejamento e controle da manutenção informatizado em uma instituição de ensino superior. Dissertação de Mestrado. UFRGS, 2007.

LAWSHE, C. H. A quantitative approach to content validity. Personnel Psychology, 28, 563-575. 1975 MARCATO, A. L. M.; FARIA, H. J.; BRANDÃO, L. E. T.; PIMENTA A.A.; SENRA, P. M. A. SILVEIRA, A.,

Um modelo para priorização da manutenção de unidades geradoras hidrelétricas utilizando a teoria das opções reais. UFSC, 2008 
MARINHA DO BRASIL. NORMAN 01 - Normas da autoridade maritima para embarcações empregadas em mar aberto. Diretoria de Portos e Costas, 2005. Disponível em: https://www.dpc.mar.mil.br. Acesso em 15/03/2014

MENEZES, H. B. Abordagem probabilistica na avaliação de desempenho da manutenção, uma experiência em telecomunicações. ABEPRO. Rio de Janeiro. RJ, 1997.

MINISTÉRIO DA AERONÁUTICA. Instrução do Comando da Aeronáutica - ICA 63-10 - Estações Prestadoras de Serviços de Telecomunicações e de Tráfego Aéreo - EPTA, 2008. Disponível em: http://publicacoes.decea.gov.br. Acesso em 12/04/2014.

MORAIS, V.C; MUHLEN, S.C. Proposta de Indicadores para Priorização de Equipamentos MédicoHospitalares em Programas de Manutenção Preventiva. UNER. Faculdad de Ingeniería de Entre Rios. Entre Rios. Argentina, 2003.

OLIVEIRA, H. M. 2013. Engenharia de Telecomunicações. 2a Edição. Recife. PE. 2013 PIRES, J. C. L.; PICCININI, M. S. Serviços de Telecomunicações: Aspectos Tecnológicos. Ensaio BNDES. Ano 1997

RANA V. S. Reliability analysis off a National Broadcasting Network (NBN) System, Microeletronics Reliability. Vol. 35. Pag.303-307. Gran Bretanha. UK, 1995.

RIBEIRO, F. J. L. Sistema de Monitoramento Subaquático para Exploração de Petróleo Usando Redes de Sensores Acústicos. Tese de doutorado; UFRJ/ COPPE/ Programa de Engenharia Elétrica; Rio de Janeiro, 2012

SAATY, T.L. How to make a decision: The analytic hierarchy process. In: European Journal of Operational Research. North-Holland: v. 48, n. 1, p. 9-26, 1991.

SILVA, A. R. N.; PEREIRA, N. C. N.; FERNANDES, G. C.; JUNIOR, M. E. Priorização da Manutenção Preventiva em Sistemas de Telecomunicações de Plataformas de Produção de Petróleo utilizando o método

AHP. CLME 2014. Inhambane. Moçambique. 2014.

SOUZA, R. D. Análise da gestão da manutenção focando a manutenção centrada na confiabilidade: estudo de caso da MRS logística. UFJF. Juiz de Fora. MG, 2008.

VELEZ, P. Industry standards result in improved offshore safety. World Oil., Vol. 224 Issue 4, p48. 2003.

WANG H. A survey of maintenance policies of deteriorating systems. European Journal of Operation Research. Vol 139. Pag. 469 a 489, 2002

WILSON, F.R.; PAN, W.; SCHUMSKY, D.A. Recalculation of the Critical Values for Lawshe's Content Validity Ratio, Measurement and Evaluation in Counseling and Development . Pag. 197 a 210. 2012

XENOS, H. G. P. Gerenciando a Manutenção Produtiva. INDG Tecnologia e Serviços - Nova Lima MG, 2004. 